\title{
Stem Cells Assisted Cancellous Bone Graft Versus Stem Cells with Demineralized Bone Matrix for Alveolar Cleft Reconstruction
}

\author{
HELMY A. SOLIMAN, M.Sc.*; HOSSAM EL-DIN A. ISMAIL, M.D.**; OMAR O. SHOUMAN, M.D.**; \\ AHMED M. BAHAAELDIN, M.D.** and MOHAMED R. EL-HADIDY, M.D.** \\ The Department of Plastic Surgery, Faculty of Medicine, Mansoura University* and Mataria Teaching Hospital**, Cairo
}

\begin{abstract}
Background: Although different bone graft materials have been suggested in the literatures for alveolar cleft reconstruction including autogenous, allogenic, xenogenic, and alloplastic grafts, Autogenous bone graft either from the iliac crest or the tibial plateau remains the gold standard against which other graft materials are evaluated. However, the procedure is invasive and associated with a potential risk of early complications such as bleeding, pain, infection, fracture and/or late complications such as chronic pain, scarring, paresthesia and gait abnormalities. Moreover, its failure rate is about $15 \%$.
\end{abstract}

Objectives: To assess the efficacy of using adipose derived stem cells (ASCs) in alveolar cleft reconstruction; whether added to the cancellous bone or used with demineralized bone matrix scaffold; in comparison to the conventional iliac crest bone grafting (ICBG).

Patients and Methods: 24 patients underwent alveolar cleft reconstruction at the age of mixed dentition over a 3years period; three of them had two grafted sites (bilateral cleft cases) giving an overall total of 27 grafted sites assessed during this study. Their mean age was 11.9 years and their mean postoperative follow-up was 11.7 months. Of these, 9 constituted the ICBG group (standard group), 10 constituted the ACSs with ICBG scaffold (ASCs/ICBG) group, whereas the remaining 8 made up ACSs with DBM (ASCs/DBM) group. Results were assessed by rating the radiographs obtained 6 months postoperatively according to Bergland scale.

Results: Alveolar cleft repairs using cancellous bone only (ICBG group) were 77.8 percent successful, alveolar cleft repairs using cancellous bone enhanced with ASCs (ASCs/ ICBG group) were 90 percent successful, and alveolar cleft repairs using DBM enhanced with ASCs (ASCs/DBM group) were 50 percent successful, but there were no significant statistical difference between the groups. ASCs/DBM group shows significantly shorter operative time, and higher cleft site infection rates.

Conclusion: Using ASCs whether with DBM or ICBG is not significantly better than the conventional method, while using DBM significantly reduced operative time, but associated with higher risk of infection.

Key Words: Alveolar cleft reconstruction - Stem cells Demineralized bone matrix.

\section{INTRODUCTION}

Alveolar cleft reconstruction is important to create a stable and continuous maxillary dental arch, facilitate closure of the oronasal fistula, improve support of teeth adjacent to the cleft site, permit further orthodontic and orthognathic interventions, and to provide support to the alar base of the nose [1]. Although different sources of bone graft materials have been suggested in the literatures for alveolar cleft reconstruction including autogenous, allogenic, xenogenic, and alloplastic grafts, autologous bone grafts either from the iliac crest or the tibial plateau remain the gold standard against which other graft materials are evaluated $[2,3]$. However, the procedure is invasive and associated with a potential risk of early complications such as bleeding, pain, infection, fracture and/or late complications such as chronic pain, scarring, paresthesia and gait abnormalities [4,5]. Moreover, its failure rate is about $15 \%$ [6]. For effective osteogenesis to take place, presence of both osteoinductive factors and osteoconductive scaffolds are needed. Cancellous bone grafts, either from iliac crest or tibial plateau display both osteoinductive and osteoconductive properties, which explain their efficacy in a wide variety of procedures [7]. On other hand, demineralized bone matrix (DBM) is an established group of allograft bone substitutes that has been used extensively in the orthopedic surgery as an osteoconductive scaffold but it has no osteoinductive potentiality [8].

ACSs act not only through direct bone formation in the gap of alveolar cleft, but also due to their paracrine effects: Production of extracellular matrix, releasing cytokines and promotion of angiogenesis $[11,12]$. ACSs in combination with a proper scaffold have a great potential that has already been proven in animal studies and on humans [11-15]. 
Our aim was to assess the efficacy of using adipose derived stem cells (ASCs) in alveolar cleft reconstruction; whether added to the cancellous bone or used with demineralized bone matrix scaffold; in comparison to the conventional cancellous bone grafting.

\section{PATIENTS AND METHODS}

After getting the approval from the Ethical Committee of Faculty of Medicine, Mansoura University, twenty-four patients with alveolar clefts were collected randomly from the outpatient clinics of the plastic surgery Centre at Mansoura University and El Mataria Teaching Hospital over a 3-years period from March of 2014 to January of 2017, three of them had two grafted sites (bilateral cleft cases) giving an overall total of 27 grafted sites assessed during this study. Their mean age was 11.9 years and their mean postoperative follow-up was 11.7 months. For each patient, age, sex, medical and surgical history was recorded. All patients were requested to have pre and 6 months post operative Periapical, occlusal, and panoramic radiograph. All patients and/or their parents were offered the three modalities of treatment, in addition to standard ICBG, they are offered the possibility of an alternative procedure using ACSs on an offlabel basis, along with autogenous ICBG or DBM as a scaffold, and the choice of a specific modality was according to each patient's (or his/her parents') preference. In bilateral cases, cancellous bone was used in one side, and cancellous bone with adipose derived stem cells was used in the other side.

Patients were then divided into three treatment groups according to the material(s) used for grafting. Of the 27 grafted sites, 9 constituted the ICBG group (standard group), 10 constituted the ACSs with ICBG scaffold (ASCs/ICBG) group, whereas the remaining 8 made up ACSs with DBM (ASCs/ DBM) group.

\section{Operative technique:}

Lidocaine $1 \%$ with epinephrine $1-200,000$ was infiltrated around the cleft margins, then intraoperative reassessment of the cleft was done as regard its extent, position of the teeth on the margins of the cleft, and presence of nasoalveolar fistula. An incision was made around the labial component of the fistula, first within the loose mucosa, then within the alveolar processes. The incision was continued along the margin of the alveolar cleft vertically toward the crest of the alveolus on each side, positioned equidistant between the labial and palatal surfaces. Once on the alveolar crest, the incisions were carried within the gingival sulci of the teeth on their labial aspect. Within the lesser segment, the incision was typically extended to the second premolar. Next, the mucoperiosteum was dissected from the alveolar processes on the labial aspect using periosteal elevator. This dissection extended to the nasal floor, exposing the lateral aspect of the anterior nasal spine and the lower pyriform rim. Through the labial approach, the mucoperiosteum was elevated off the bony walls of the cleft from the alveolar crest to the nasal floor. The oronasal fistula tract was then dissected and closed using interrupted 4/0 Vicryl (Ethicon, Inc., Somerville, N.J.) sutures. At this time, before placement of the bone graft, adequate labial soft tissue mobility that will provide a tension-free closure over the bone graft was confirmed. If greater mobility was needed, horizontal scoring of the periosteum at the base of the lesser segment flap was done. Extending the back cut and directing it anteriorly gained further mobility if needed. After introducing the graft material(s), the lesser segment mucoperiosteal flap was advanced medially and slightly palatally to cover the bone graft and to provide the oral labial closure of the fistula using interrupted 4/0 Vicryl sutures. Interrupted interdental papilla sutures were then placed to stabilize the labial and palatal tissues toward one another and against the bony alveolar process.

\section{Harvesting cancellous bone from the iliac crest:}

The Cancellous Bone is harvested from the Iliac Crest using the standard technique, taking into consideration not to place the scar on the bone prominence. $(1 \mathrm{~cm}$ posterior and lateral to anterior superior iliac spine. $3 \mathrm{cc}$ of the tumescent solution was injected at the incision site; incision was then taken by 15 blade through the skin and continued by diathermy down to the periosteum. Incision was then taken through the periosteum to expose the bone, and an osteotome was used to make a trap door fenestration (two vertical cuts and one from the medial aspect of the crest to connect the vertical cuts), then an anteriorly based cortical bone flap was elevated to expose the cancellous bone. A curette was then used to extract as much cancellous bone as needed. The cortical roof was then reduced back in place to cover the donor site and reduce the postoperative bleeding. If there were any defects or fragmentation of the cortex of the roof, bone wax was used to seal the cancellous bone cavity. Vicryl 3/0 sutures were used for the re-attachment of the muscles and for closure of the subcutaneous layer, and Monocryl 4/0 (Ethicon, Inc., Somerville, N.J.) sutures were used in a subQ pattern to close the skin. 1 $\mathrm{cc}$ of Xylocaine 1\% 
+1 cc of Marcaine $0.25 \%$ was injected to relief postoperative pain and soft dressing was applied.

Preparation of the demineralized bone matrix (in ASCS/DBM group):

Using sterile technique on a side table with gloves, the demineralized bone matrix powder (Wright medical technology, Inc., Arlington, TN) was emptied into the mixing bowl $(5 \mathrm{cc})$. The mixing solution was then emptied into the bowl gradually and mixed with spatula and the material kneaded against the sidewall of the bowl until the desired consistency was achieved (approximately 30-60 seconds). After achieving a putty-like consistency, the material can be handled digitally. Material maintains handling characteristics up to 10 minutes after mixing, during that period it was implanted in the already prepared cleft gap.

Preparation of adipose derived stem cells from the fat:

Fat grafts were harvested at the end of the procedure. Donner site was the lower abdomen in all cases. In ACSs/ICBG group, the aspiration cannula was introduced through the same incision of bone graft harvesting to avoid additional scarring. In ACSs/DBM group, the aspiration cannula was introduced through a small $0.5 \mathrm{~cm}$ stab incision in the umbilicus at 6 O'clock. Liposuction was performed as described by SR Coleman [16]. A long a traumatic 3-mm Mercedes cannula (luer lock type) was used. An average amount of $60 \mathrm{cc}$ was usually harvested (range $50-80 \mathrm{cc}$ ). The raw aspirate was then collected for stem cell preparation. Fat was extensively washed with sterile phosphate buffered saline (PBS) to remove the blood cells, saline, and local anesthetics. Then ACSs were separated from adipose tissue by cell digestion using $0.075 \%$ collagenase type I solution (Collagenase NB4 Standard, SERVA Electrophoresis, Heidelberg, Germany) at $37^{\circ} \mathrm{C}$ for $30 \mathrm{~min}$ to one hour. An equal volume of Dulbecco's modified Eagle medium (DMEM) containing 10\% fetal bovine serum (FBS) and 1\% penicillin-streptomycin (Gibco, Carlsbad, CA, USA) was then added to inactivate collagenase. The digest was centrifuged at $1,500 \mathrm{rpm}$ for $5 \mathrm{~min}$. The supernatant was removed, and the cell pellet, termed the Stromal Vascular fraction (SVF), was left. The SVF, containing ASCs, was resuspended in 10\% FBS then recentrifuged and filtered through a 100-lm nylon filter. The cell pellet was resuspended in a $10 \mathrm{ml}$ complete culture medium formed of DMEM, $13 \%$ FBS and $1.5 \%$ Penicillin streptomycin mixture (Lonza, Verviers, Belgium). The cell suspension was cultured in culture flask $25 \mathrm{~cm}^{2}$ (Easy Flask,
Nunc, Roskilde, Denmark) and incubated in $\mathrm{CO} 2$ incubator (Nuaire, NU 4950E, Autoflow Water Jacketed CO2 incubator, USA) at $37^{\circ} \mathrm{c}$ and $5 \%$ $\mathrm{CO} 2$. The ASCs were prepared in a final concentration of $3 \times 106 / \mathrm{ml}$ and its identity was confirmed by flow cytometry (positive for CD49, CD71, CD73, CD90, CD105 and negative for CD31, CD34, CD45), and then supplied in a tube for injection.

\section{Post operative follow-up:}

All patients instructed to have clear fluids only for the first 48 hours after surgery, then soft diet for 4 weeks. Antibiotics prophylaxis was given before surgery, and for 3 days after surgery. Mouthwash was used regularly after meals for one week. All patients were stable and discharged from the hospital in the second postoperative day. Followup visit was scheduled in the outpatient clinic one week after surgery when checking of the oral wound and dressing of the graft donner site (in ICBG and ASCs/ICBG groups) were done. Prepared ASCs was injected in the pocket of the bone graft at that time using a 27-gauge needle (for ASCs/ICBG and ASCs/DBM groups). By that time the mucosa was already healed and the pocket is sealed.

\section{Radiographic outcomes:}

In this study, the success of autogenous bone grafts was assessed through evaluating the radiographs taken at 6 months postoperatively using the indicators of surgical success described by Bergland [17] (Fig. 1). Bone grafts of types I and II according to the Bergland scale were considered successful bone grafts, whereas the other types were considered unsuccessful.

\section{Statistical analysis:}

Graph Pad Prism Version 5 for Mac (GraphPad Software, Inc., La Jolla, Calif.) was used to carry out statistical tests. The Fisher Exact Test was used to analyze the data comparing the success rate, the complications rate and the operative time between the three groups. $p$-value was regarded as significant if less than 0.05 .

\section{RESULTS}

This study included twenty-four patients; three of them had two grafted sites (bilateral cleft cases) giving an overall total of 27 grafted sites assessed during this study; male predominance was observed in this study as fifteen patients were males (62.5\%) and nine were females (37.5\%), with male to female ration of 1.6:1. Mean age of the patients at the 
time of surgery was 11.9 years (range 7.5-27 years). Mean follow-up period was 11.7 months (range 624 months) (Table 1). Twenty patients were suffering from unilateral alveolar cleft (83.4\%), and four patients were suffering from bilateral alveolar cleft $(16.6 \%)$. Of the twenty cases with unilateral clefts, 13 were on the left side (65\%), and 7 cases were of the right side $(35 \%)$. So the overall distribution of the cases according to the side affected was 13 cases were unilateral on the left side (54\%), 7 cases were unilateral on the right side (29\%) and 4 cases were bilateral $(17 \%)$.

Twenty-one patient were primary cases (not operated before) $(87.5 \%)$, and three patients were operated before and needed revision (12.5) due to either previous failure or inadequate bone formation; of these three two were unilateral and one was bilateral that needed revision of the right side only. Fifteen patients had presurgical orthodontic preparation (62.5\%) for an average of 6.3 months (range 3-9 months), and nine patients did not have presurgical orthodontic preparation (37.5\%).
There were no significant differences between the groups with respect to number of patients, age, sex, laterality of the cleft and whether the surgery was primary or revisional.

Alveolar cleft repairs using cancellous bone only were 77.8 percent successful, alveolar cleft repairs using cancellous bone enhanced with ASCs were 90 percent successful, and alveolar cleft repairs using DBM enhanced with ASCs were 50 percent successful. But there were no significant statistical difference between the groups ( $p$-value $>0.05$ ) (Table 2) (Fig. 2).

Operative time in group $\mathrm{C}$ was significantly shorter than in the other two groups, representing a time savings of 92 minutes per case, on average. (Fig. 3).

The over all postoperative complications such as infection, wound dehiscence, graft resorption and donner site complications were highest in group $\mathrm{C}$ patients and lowest in group B patients with no statistical significance ( $p$-value $>0.05)$. (Fig. 4).

Table (1): Comparison between all groups according to different parameters.

\begin{tabular}{|c|c|c|c|c|c|c|c|c|}
\hline & \multirow{2}{*}{\multicolumn{2}{|c|}{$\begin{array}{c}\text { ICBG } \\
\text { group }\end{array}$}} & \multirow{2}{*}{\multicolumn{2}{|c|}{$\begin{array}{c}\begin{array}{c}\mathrm{ASCs} / \mathrm{ICBG} \\
\text { group }\end{array} \\
\mathrm{N}=10 \\
\end{array}$}} & \multirow{2}{*}{\multicolumn{2}{|c|}{$\begin{array}{c}\begin{array}{c}\text { ASCs/DBM } \\
\text { group }\end{array} \\
\mathrm{N}=8 \\
\end{array}$}} & \multicolumn{2}{|c|}{$\begin{array}{l}\text { Chi-square } \\
\text { test }\end{array}$} \\
\hline & & & & & & & \multirow{2}{*}{$X^{2}$} & \multirow{2}{*}{$p$-value } \\
\hline & No. & $\%$ & No. & $\%$ & No. & $\%$ & & \\
\hline \multicolumn{9}{|l|}{ Age (y): } \\
\hline $7.5-12 y$ & 7 & 77.8 & 8 & 80.0 & 6 & 75.0 & \multirow{3}{*}{0.064} & \multirow{3}{*}{0.999} \\
\hline$>12-18 y$ & 1 & 11.1 & 1 & 10.0 & 1 & 12.5 & & \\
\hline$>18 \mathrm{y}$ & 1 & 11.1 & 1 & 10.0 & 1 & 12.5 & & \\
\hline \multicolumn{9}{|l|}{ Sex: } \\
\hline Males & 6 & 66.7 & 6 & 60.0 & 4 & 50.0 & \multirow{2}{*}{0.491} & \multirow{2}{*}{0.782} \\
\hline Females & 3 & 33.3 & 4 & 40.0 & 4 & 50.0 & & \\
\hline \multicolumn{9}{|l|}{ Laterality: } \\
\hline Rt unilateral & 3 & 33.3 & 2 & 20.0 & 2 & 25.0 & \multirow{3}{*}{1.899} & \multirow{3}{*}{0.754} \\
\hline Lt unilateral & 3 & 33.3 & 5 & 50.0 & 5 & 62.5 & & \\
\hline Bilateral & 3 & 33.3 & 3 & 30.0 & 1 & 12.5 & & \\
\hline \multicolumn{9}{|l|}{ Primary or Revision: } \\
\hline Primary & 8 & 88.9 & 9 & 90.0 & 7 & 87.5 & \multirow{2}{*}{0.028} & \multirow{2}{*}{0.986} \\
\hline Revision & 1 & 11.1 & 1 & 10.0 & 1 & 12.5 & & \\
\hline Operative time (min) & \multicolumn{2}{|c|}{$195 \pm 35$} & \multicolumn{2}{|c|}{$188 \pm 40$} & \multicolumn{2}{|c|}{$110 \pm 20$} & & $<0.05^{*}$ \\
\hline \multicolumn{9}{|l|}{ Bergland Score: } \\
\hline Type I & 5 & 55.6 & 6 & 60.0 & 2 & 25.0 & \multirow{4}{*}{6.789} & \multirow{4}{*}{0.34} \\
\hline Type II & 2 & 22.2 & 3 & 30.0 & 2 & 25.0 & & \\
\hline Type III & 2 & 22.2 & 1 & 10.0 & 2 & 25.0 & & \\
\hline Type IV & 0 & 0.0 & 0 & 0.0 & 2 & 25.0 & & \\
\hline \multicolumn{9}{|l|}{ Complications: } \\
\hline Infection & 0 & 0.0 & 0 & 0.0 & 2 & 25.0 & \multirow{4}{*}{6.735} & \multirow{4}{*}{0.346} \\
\hline Dehiscence & 0 & 0.0 & 0 & 0.0 & 1 & 12.5 & & \\
\hline $\begin{array}{l}\text { Donner site } \\
\text { Complications }\end{array}$ & 0 & 0.0 & 1 & 10.0 & 0 & 0.0 & & \\
\hline Graft resorption & 2 & 22.2 & 1 & 10.0 & 4 & 50.0 & & \\
\hline
\end{tabular}


Table (2): Comparison between groups according to success of bone grafts.

\begin{tabular}{|c|c|c|c|c|c|c|c|c|}
\hline \multirow[t]{2}{*}{ Results } & \multicolumn{2}{|c|}{$\begin{array}{l}\text { ICBG } \\
\text { group }\end{array}$} & \multicolumn{2}{|c|}{$\begin{array}{c}\text { ASCs/ICBG } \\
\text { group }\end{array}$} & \multicolumn{2}{|c|}{$\begin{array}{c}\text { ASCs/DBM } \\
\text { group }\end{array}$} & \multicolumn{2}{|c|}{$\begin{array}{c}\text { Chi-square } \\
\text { test }\end{array}$} \\
\hline & No. & $\%$ & No. & $\%$ & No. & $\%$ & $X^{2}$ & $p$-value \\
\hline Success & 7 & 77.8 & 9 & 90.0 & 4 & 50.0 & & \\
\hline Failure & 2 & 22.2 & 1 & 10.0 & 4 & 50.0 & 3.799 & 0.150 \\
\hline Total & 9 & 100.0 & 10 & 100.0 & 8 & 100.0 & & \\
\hline
\end{tabular}

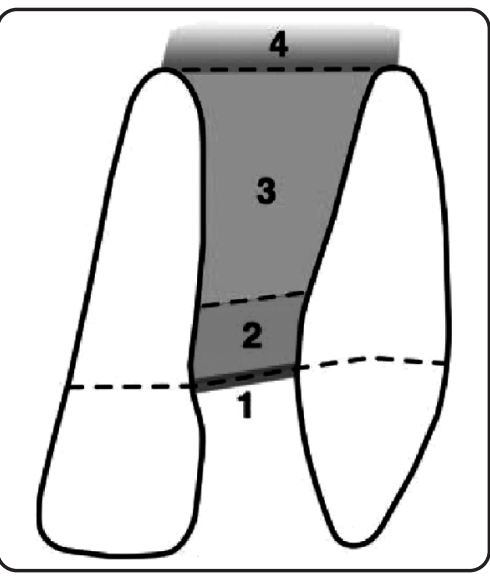

Fig. (1): Bergland scale17: The amount of bone produced in the cleft site was evaluated based on the height of the intraalveolar septum (Bone Bridge). Heights are measured from the apical extent of the cleft site (a line between the tips of the roots of the adjacent teeth) to the cementoenamel junction coronally. Type 1 is defined as normal height (more than $75 \%$ of the normal bone height). Type 2 is defined as less than normal height (50-75\% of normal bone height). Type 3 has less than $50 \%$ of normal bone height. Type 4 has no bone bridge in the cleft site.

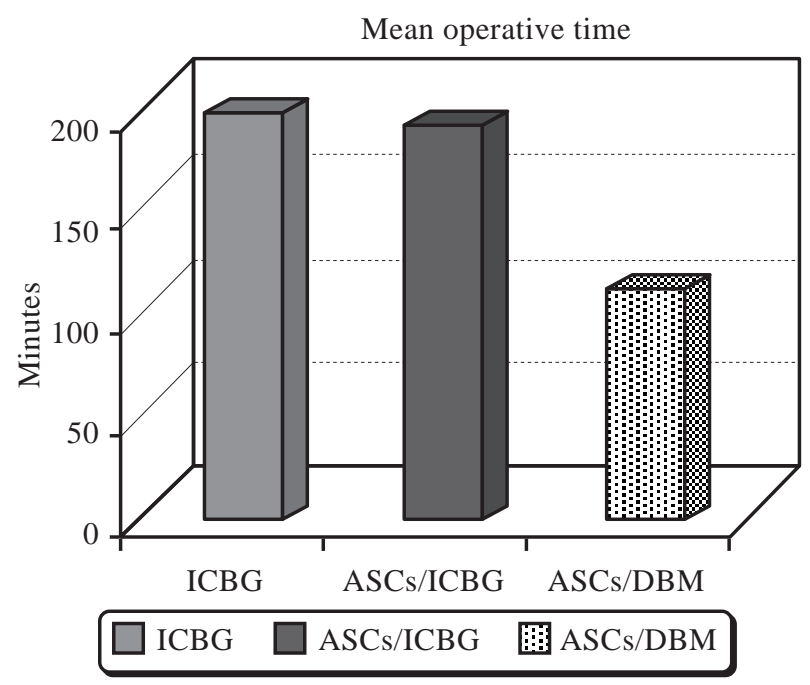

Fig. (3): Histogram shows mean operative times in the groups.

\section{DISCUSSION}

Alveolar cleft reconstruction as a component of the cleft lip and palate management; although being performed very widely; has been one of the

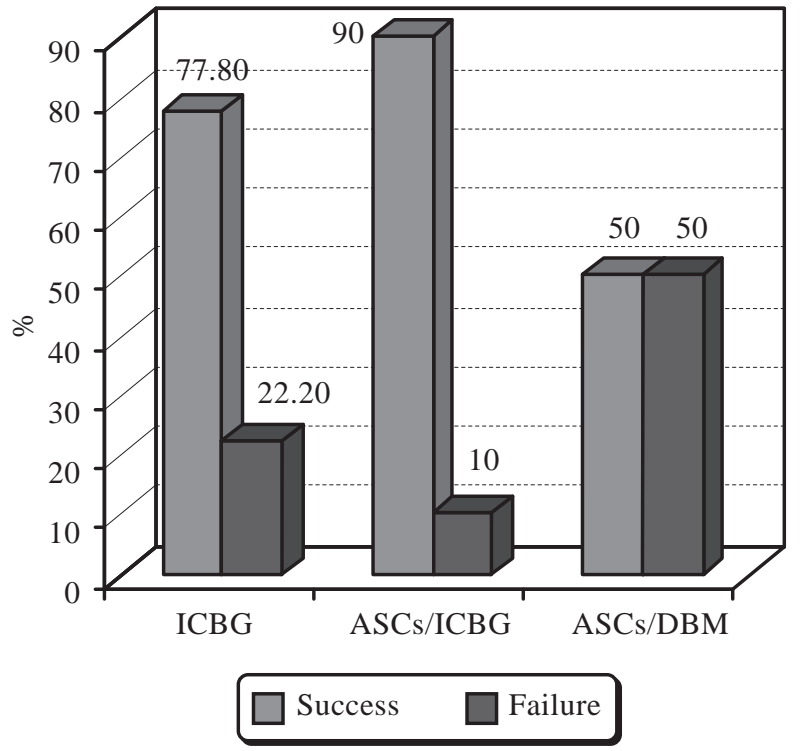

Fig. (2): Histogram shows comparison between groups according to success of bone grafts.

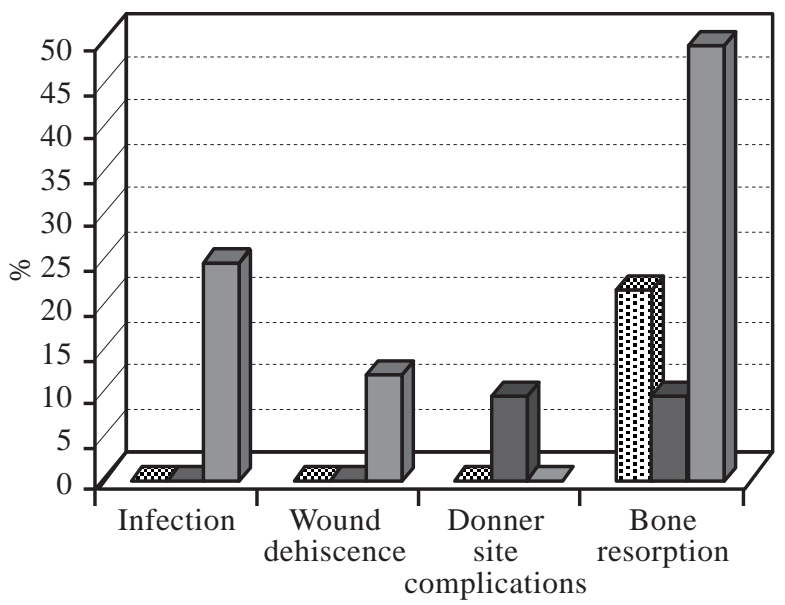

臯 ICBG $\square$ ASCs/ICBG $\square$ ASCs/DBM

Fig. (4): Histogram shows comparison between groups according the rate of complications.

most controversial surgical procedures since it began in early $20^{\text {th }}$ century till now. There are multiple philosophies and treatment modalities regarding each step in alveolar cleft diagnosis and management including the most appropriate age, 
the ideal grafting material, method of evaluation and whether adjunctive procedures such as orthodontic treatment should be used before or after grafting [1]. Despite many evidences to support the superiority of secondary alveolar bone grafting using ICBG, and despite being the commonest graft used, and the standardized graft to which different types of alveolar bone grafts should be compared [18], two major disadvantages remain. First, its associated donor-site morbidities including delayed ambulation, pain, hematoma, discomfort, nerve injury, prolonged hospitalization, and limited volume that can be harvested, second, failure rate is about $15 \%$ [7]. So, in an effort to reduce the donner site morbidity, many other sources of bone graft have been suggested in the literature for alveolar cleft reconstruction including allogenic, xenogenic, and alloplastic grafts. The outcomes achieved with different graft materials have been extensively studied and compared in the literatures, and each type has its advantages and disadvantages [1]. But the ideal bone graft material remains controversial till now [19].

Considering these complications, limitations, together with other problems associated with $\mathrm{CL} / \mathrm{P}$ patients, alternative approaches to spare donner site and to improve outcome are needed. So, we developed our technique of using adipose derived stem cells with demineralized bone matrix (allograft) scaffold (in group "C" patients) as an alternative to the autogenous bone grafting assuming that: The osteogenic properties of ASCs and the osteoconductive scaffolds (DBM) will replicate the properties of autogenous bone, without the need for graft harvesting procedure [20]. We also used ACS s with ICBG in group "B" patients to examine whether this will improve results or not in comparison to the conventional ICBG.

In the present study, twenty-four patients (with twenty seven grafted sites) were chosen randomly and divided into three treatment groups. ICBG group, in which we used cancellous bone only; ASCs/ICBG group, in which ACSs with cancellous bone were used; and ASCs/DBM group, in which we used demineralized bone matrix with ACSs.

We searched the literature for the similar studies that applied ASCs on either cancellous bone or DBM till the time of launching our study, and to the best of our knowledge, this is the first prospective study that used either the demineralized bone matrix or cancellous bone as a scaffold for adipose derived stem cells for alveolar cleft reconstruction.

In this study we chose adipose tissue as a source for the stem cells similar to Gimble et al., Zuk et al., and Yoshimura et al. [4,21,22]. Others like Hibi et al., Pradel et al., and Behnia et al., used mesenchymal stem cells from the bone marrow $[\mathbf{1 4 , 1 5 , 2 3 ]}$. The advantage of adipose tissue is that, it represents an abundant, reliable, noninvasive, and accessible source of adult stem cells. Lipoaspirate provides an easily obtainable source of ASCs at a frequency of 1:100 to $1: 1500$ cells. This greatly exceeds the frequency of mesenchymal stem cells (MSCs) from bone marrow 500 -fold, with $1 \mathrm{~g}$ of adipose tissue contains nearly 5,000 ASCs [24,25].

In this study, the preparation of ASCs was done following $\mathrm{Lu}$ et al with some modification where fat was digested with $0.2 \%$ collagenase for 50 minutes instead of $0.1 \%$ collagenase for 40 minutes [26]. We confirmed that ASCs express characteristic surface markers by flow cytometry (positive for CD36, CD49, CD71, CD73, CD90, CD105 and negative for CD31, CD34, CD45). It has been proven in the literatures that ACSs have the capability of differentiation to different cell lines including osteocytes $[\mathbf{4 , 2 1}$. Guasti et al., studied ACSs harvested from abdominal fat in pediatrics, and they proved its high capability of differentiation to osteogenic tissue [27].

Different materials were found to be successful scaffolds for ACSs such as collagen, titanium, fibrin glue, and betatricalcium phosphate (B-TCP) [28-31]. Hibi et al., used MSCs obtained from bone marrow with titanium scaffold in a child with alveolar cleft. The cells were cultured then differentiated into osteogenic tissue that resulted in regeneration of $79 \%$ of bone with successful eruption of canine and lateral incisor [23]. Pradel et al., showed complete defect closure and tooth eruption after filling the alveolar cleft defect with MSCs harvested from the maxilla in a bovine collagen matrix scaffold [14]. Behnia et al., utilized MSCs from the bone marrow carried on a scaffold that combined demineralized bone and calcium sulfate for alveolar cleft reconstruction. The results suggested that the amount of bone formation was inadequate and indicated that the conventional bone substitute was a favorable scaffold for MSCs for alveolar bone regeneration [15].

In the present study, we used demineralized bone matrix (DBM) as an osteoconductive scaffold for alveolar cleft reconstruction in ASCs/DBM group similar to Cameron et al. [32], Behnia et al. [15], Sivak et al. [33], Macisaac et al. [34] and Louis et al. [35]. We used the ICBG in ICBG and ASCs/ ICBG groups similar to Kom et al. [36], Behnia et al. [15], and Yuanzheng et al. [37] who enhanced the autologous iliac bone with MSCs from the iliac 
crest. Others like Benliday et al., used bovine hydroxiapetite [38], Pradel et al., used bovine collagen matrix [14], and De Ruiter et al., used tricalcium phosphate [39]. DBM has the advantages of being osteoconductive, it doesn't cause local foreign-body immunogenic reaction as the antigenic surface structure of the bone is destroyed during demineralization, its degradation doesn't produce any products that affect new bone formation, and being prepared by acid extraction of allografts, it retains collagen and other proteins. Although transmission of diseases has not yet been reported with DBM but is theoretically possible [40].

Many osteoinductive factors have been used in the literatures to enhance either cancellous bone or DBM to improve bone formation. The most frequently used are Platelet rich plasma and Bone morphogenic proteins $[32,41,42]$ Backly et al., proved that platelets rich plasma enhances osteogenesis through its osteoinductive effects attributed to the secreted growth factors [43]. Dutra et al., combined platelets rich plasma with glass foam scaffold to reconstruct alveolar clefts and obtain good results [44]. In contrast, Luaces et al., found no significant advantages of adding PRP to ICBG over the ICBG alone in 20 patients underwent alveoloplasty [45].

Cameron et al., compared the results of using DBM enhanced with BMPs with the conventional ICBG and they found excellent results in the group that used DBM with BMPs with success rate of $97.2 \%$ compared to $84.2 \%$ for the group that used ICBG, with significant decrease in the operative time [32]. Canan et al., and Fallucco et al., used BMP in alveolar cleft reconstruction with results comparable to autologous ICBG [46,47]. Others as Neovius et al., had to terminate their study due to severe gingivitis in patients receiving BMP [48].

In this study, a statistically non-significant difference was detected in the success rates between the three groups $\left(\right.$ Chi X $\mathrm{X}^{2}=3.799, p$-value $\left.=0.150\right)$. We also did not find significant difference between the groups as regard the rate of complications (Chi $\mathrm{X}^{2}=6.735, p$-value $\left.=0.346\right)$. But we found a significant shorter operative time in group " $C$ " as compared to the other two groups ( $p$-value $<0.05$ ), representing a time saving of 92 minutes per case, on average. This is similar to Cameron et al., who found significant shorter operative time when DBM was used [32].

In ICBG group (cancellous bone only was used), success rate was $77.8 \%$, and failure rate was $22.2 \%$. These results are better than Dickinson et al., who reported 63\% success rate in the control group [49].
Also better than Ananth et al who reported 69\% success rate in unilateral clefts [50]. But less than Cameron et al., who reported success rate of $84.2 \%$ [32], and also less than Felstead et al., who reported success rate of $94 \%$ [51]. In ASCs/ICBG group (cancellous bone with ASCs was used), success rate was 90\%, and in ASCs/DBM group (DBM with ASCs was used), success rate was $50 \%$. As long as no studies in literatures were found using ACSs with either DBM or ICBG scaffold, we compared only results of ICBG group with similar studies but we could not compare results of ASCs/ICBG or ASCs/DBM groups with other studies.

The over all postoperative complications in this study were infection (25\%), wound dehiscence $(12.5 \%)$, graft resorption (18.5\%) and donner site complications (10\%), and these are similar to the percentages in most of the literatures. Comparison between groups; considering the complication rates, showed no significant differences. The limitation of our study was the small number of the patient in each group that might be the reason for non-significant results.

\section{REFERENCES}

1- Horswell B.B. and Henderson J.M.: Secondary osteoplasty of the alveolar cleft defect. J. Oral Maxillofac. Surg., 61 (9): 1082-1090, 2003.

2- Jia Y.L., Fu M.K. and Ma L.: Long-term outcome of secondary alveolar bone grafting in patients with various types of cleft. Br. J. Oral Maxillofac. Surg., 44: 308-312, 2006.

3- Cohen M., Figueroa A.A., Haviv Y., et al.: Iliac versus cranial bone for secondary grafting of residual alveolar clefts. Plast Reconstr Surg., 87: 423-427, 1991.

4- Gimbel M., Ashley R.K., Sisodia M., et al.: Repair of alveolar cleft defects: Reduced morbidity with bone marrow stem cells in a resorbable matrix. J. Craniofac. Surg., 18 (4): 895-901, 2007.

5- Moreau J.L., Caccamese J.F., Coletti D.P., et al.: Tissue engineering solutions for cleft palates. J. Oral Maxillofac. Surg., 65 (12): 2503-11, 2007.

6- Schultze-Mosgau S., Nkenke E., Schlegel A.K., et al.: Analysis of bone resorption after secondary alveolar cleft bone grafts before and after canine eruption in connection with orthodontic gap closure or prosthodontic treatment. J. Oral Maxillofac. Surg., 61 (11): 1245-1253, 2003.

7- Viktot T., Zach J., Maryam K., et al.: Stem cells, growth factors and scaffolds in craniofacial regenerative medicine. Genes \& Diseases J., 3 (1): 56-71, 2016.

8- Filho O., Ozawa T., Bachega C., et al.: Reconstruction of alveolar cleft with allogenous bone graft: Clinical considerations. Dent. Press J. Orthod, 18 (6): 138-147, 2013.

9- Tanimoto K., Sumi K., Yoshioka M., et al.: Experimental tooth movement into new bone area regenerated by use of bone marrow-derived mesenchymal stem cells. Cleft 
Palate Craniofac. J., 19: doi: http://dx.doi.org/10.1597/12232, 2013.

10- Zhang D., Chu F., Yang Y., et al.: Orthodontic tooth movement in alveolar cleft repaired with a tissue engineering bone: An experimental study in dogs. Tissue Eng. Part A, 17 (9-10): 1313-1325, 2011.

11- Pourebrahim N., Hashemibeni B., Shahnaseri S., et al.: A comparison of tissue-engineered bone from adiposederived stem cell with autogenous bone repair in maxillary alveolar cleft model in dogs. Int. J. Oral Maxillofac. Surg., 42 (5): 562-570, 2013.

12- Korn P., Schulz M.C., Range U., et al.: Efficacy of tissue engineered bone grafts containing mesenchymal stromal cells for cleft alveolar osteoplasty in a rat model. J. Craniomaxillofac. Surg., 4. pii: S1010 5182(14)00090$0,2014$.

13- Pradel W., Tausche E., Gollogly J., et al.: Spontaneous tooth eruption after alveolar cleft osteoplasty using tissueengineered bone: A case report. Oral Surg. Oral Med. Oral Pathol. Oral Radiol. Endod J., 105 (4): 440-444, 2008.

14- Behnia H., Khojasteh A., Soleimani M., et al.: Repair of alveolar cleft defect with mesenchymal stem cells and platelet derived growth factors: A preliminary report. J. Craniomaxillofac. Surg., 40 (1): 2-7, 2012.

15- Stanko P., Mracna J., Stebel A., et al.: Mesenchymal stem cells - a promising perspective in the orofacial cleft surgery. Bratisl Lek Listy, 114 (2): 50-52, 2013.

16- Coleman S.R.: Structural fat grafts: The ideal filler?, Clin. Plast. Surg., 28: 111e9, 2001.

17- Bergland O., Semb G., abyholm F., et al.: Secondary bone grafting and orthodontic treatment in patients with bilateral complete clefts of the lip. Annals of Plastic Surgery J., 17 (6): 460-474, 1986.

18- Rawashdeh M. and Telfah H.: Secondary alveolar bone grafting: The dilemma of donor site selection and morbidity. Br. J. Oral Maxillofac. Surg., 46 (8): 665-670, 2008.

19- Ochs M.: Alveolar cleft bone grafting (Part II): Secondary bone grafting. J. Oral Maxillofac. Surg., 54 (1): 83-88, 1996.

20- Oppenheimer A.J., Mesa J. and Buchman S.R.: Current and emerging basic science concepts in bone biology: implications in craniofacial surgery. Craniofac. Surg. J., 23: 30-36, 2012.

21- Zuk P., Zhu M., Mizuno H., et al.: Multilineage cells from human adipose tissue: Implications for cell-based therapies. Tissue Eng. J., 7: 211-218, 2001.

22- Yoshimura K., Sato K., Aoi N., et al.: Cell-assisted lipotransfer for cosmetic breast augmentation: Supportive use of adipose-derived stem/stromal cells. Aesthetic Plast. Surg. J., 32: 48-55, 2008.

23- Hibi H., Yamada Y., Ueda M., et al.: Alveolar cleft osteoplasty using tissue-engineered osteogenic material. Int. J. Oral Maxillofac. Surg., 35 (6): 551-555, 2006.

24- Peng L., Jia Z., Yin X., et al.: Comparative Analysis of Mesenchymal Stem Cells from Bone Marrow, Cartilage, and Adipose Tissue. Stem Cells and Development J., 17: 761-774, 2008
25- Rubio D., Garcia-Castro J., Bernad A., et al.: Spontaneous human adult stem cell transformation. Cancer Res. J., 65 (8): 3035-3039, 2005.

26- Lu F., Mizuno H., Uyasal C., et al.: Improved viability of random pattern skin flaps through the use of adipose derived stem cells. Plast. Reconstr. Surg. J., 121: 50-58, 2008.

27- Guasti L., Prasongchean W., Kleftouris G., et al.: High plasticity of pediatric adipose tissue derived stem cells: Too much for selective skeletogenic differentiation? Stem Cells Transl Med., 1 (5): 384-395, 2012.

28- Yang P., Huang X., Wang C., et al.: Repair of bone defects using a new biomimetic construction fabricated by adiposederived stem cells, collagen I, and porous beta-tricalcium phosphate scaffolds. Exp. Biol. Med. (Maywood), 238 (12): 1331-1343, 2013.

29-. Daei-Farshbaf N., Ardeshirylajimi A., Seyedjafari E., et al.: Bioceramic-collagen scaffolds loaded with human adipose-tissue derived stem cells for bone tissue engineering, 41 (2): 741-749, 2014.

30- Benazzo F., Botta L., Scaffino M.F., et al.: Trabecular titanium can induce in vitro osteogenic differentiation of human adipose derived stem cells without osteogenic factors. J. Biomed Mater Res. A, 102 (7): 2061-2071, 2014.

31- Lendeckel S., Jödicke A., Christophis P., et al.: Autologous stem cells (adipose) and fibrin glue used to treat widespread traumatic calvarial defects: Case report. J. Craniomaxillofac. Surg., 32 (6): 370-373, 2004.

32- Cameron S., Francis M., Sheila S., et al.: rhBMP-2 with a demineralized bone matrix scaffold versus autologous iliac crest bone graft for alveolar cleft reconstruction. Plast. Reconstr. Surg. J., 131 (5): 1107-1115, 2013.

33- Sivak W., Macisaac Z., Rottgers S., et al.: Management of failed alveolar bone grafts: Improved outcomes and decreased morbidity with allograft alone. Plast. Reconstr. Surg. J., 133: 345-354, 2014.

34- Macisaac Z., Rottgers S., Davit A., et al.: Alveolar reconstruction in cleft patients: decreased morbidity and improved outcomes with supplemental demineralized bone matrix and cancellous allograft. Plast. Reconstr. Surg. J., 130: 625-632, 2012.

35- Louis J., Murakami H., Kim H., et al.: Evidence of osteoinduction by Grafton demineralized bone matrix in nonhuman primate spinal fusion. Spine J., 29: 360-366, 2004.

36- Kom P., Schulz M., Range U., et al.: Efficacy of tissue engineered bone grafts containing mesenchymal stromal cells for cleft alveolar osteoplasty in a rat model. J. Craniomaxillofac. Surg., 42 (7): 1277-1285, 2014.

37- Yuanzheng C., Yan G., Ting L., et al.: Enhancement of the repair of dog alveolar cleft by an autologous iliac bone, bone marrow-derived mesenchymal stem cell, and platelet-rich fibrin mixture. Plast. Reconstr. Surg. J., 135 (5): 1404-1427, 2015.

38- Benliday, Tatil U., Kurkcu M., et al.: Comparison of bovine-derived hydroxyapatite and autogenous bone for secondary alveolar bone grafting in patients with alveolar clefts. Oral Maxillofac. Surg. J., 1: 95-102, 2012. 
39- De Ruiter A., Janssen N., van Es R., et al.: Micro structured beta-tricalcium phosphate for repair of the alveolar cleft in cleft lip and palate patients: A pilot study. Cleft Palate Craniofac. J., 52 (3): 336-340, 2015.

40- Tuli S.M. and Singh A.D.: The osteoninductive property of decalcified bone matrix. An experimental study. J. Bone Joint Surg., 60: 116-123, 1978.

41- Marukawa E., Oshina H., Iino G., et al.: Reduction of bone resorption by the application of platelet-rich plasma (PRP) in bone grafting of the alveolar cleft. J. Craniomaxillofac. Surg., 39 (4): 278-283, 2011.

42- Alonso N., Tanikawa D.Y., Freitas Rda S., et al.: Evaluation of maxillary alveolar reconstruction using a resorbable collagen sponge with recombinant human bone morphogenetic protein-2 in cleft lip and palate patients. Tissue Eng. Part C Methods, 16 (5): 1183-1192, 2010.

43- Backly R.M., Zaky S.H., Canciani B., et al.: Platelet rich plasma enhances osteoconductive properties of a hydroxyapatite- $\beta$-tricalcium phosphate scaffold (Skelite) for late healing of critical size rabbit calvarial defects. J. Craniomaxillofac. Surg., 42 (5e): 70-79, 2014.

44- Dutra C.E., Pereira M.M., Serakides R., et al.: In vivo evaluation of bioactive glass foams associated with platelet-rich plasma in bone defects. J. Tissue Eng. Regen. Med., 2 (4): 221-228, 2008

45- Luaces-Rey R., Arenaz-Búa J., Lopez-Cedrún-Cembranos J.L., et al.: Is PRP useful in alveolar cleft reconstruction?
Platelet-rich plasma in secondary alveoloplasty. Med. Oral Patol. Oral Cir. Bucal, 15 (4e): 619-623, 2010.

46- Canan L.W., da Silva Freitas R., Alonso N., et al.: Human bone morphogenetic protein-2 use for maxillary reconstruction in cleft lip and palate patients. J. Craniofac. Surg., 23 (6): 1627-1633, 2012.

47- Fallucco M.A. and Carstens M.H.: Primary reconstruction of alveolar clefts using recombinant human bone morphogenic protein-2: Clinical and radiographic outcomes. J. Craniofac. Surg., 2: 1759-1764, 2009.

48- Neovius E., Lemberger M., Docherty A.C., et al.: Alveolar bone healing accompanied by severe swelling in cleft children treated with bone morphogenetic protein-2 delivered by hydrogel. J. Plast. Reconstr. Aesthet. Surg., 66 (1): 37-42, 2013.

49- Dickinson B., Ashley B., Wasson R., et al.: Reduced morbidity and improved healing with bone morphogenic protein-2 in older patients with alveolar cleft defects. Plast. Reconstr. Surg. J., 121 (1): 209-217, 2008.

50- Ananth S., Murthy M., James A., et al.: Secondary alveolar bone grafting: An outcome analysis. Can J. Plast. Surg., 14 (3): 172-174, 2006.

51- Felstead A.M., Deacom S. and Revington P.: The outcome for secondary alveolar bone grafting in the South West UK region post-CSAG. Cleft Palate Craniofac. J., 47 (4): 359-362, 2010 